\title{
Ergodic polynomials on 2-adic spheres
}

by

\author{
Nacima MEMIĆ \\ Presented by Feliks PRZYTYCKI
}

Summary. We provide sufficient conditions for invariance, isometricity and ergodicity of polynomials on 2-adic spheres. These conditions are described as relations satisfied by 2-adic coefficients.

1. Introduction. It is known that rational functions are not ergodic on the infinite measure set of $p$-adic numbers [7]. In the same paper it was proved that any invertible measure-preserving rational function must be invariant on sufficiently large spheres.

In this work we study the behaviour of polynomials which are invariant on spheres of 2-adic numbers. We establish sufficient conditions for their ergodicity on these compact sets described by their 2-adic coefficients. Polynomials having 2-adic coefficients were studied in [4, [8] and [11. These are obviously invariant and measure-preserving on the set of 2-adic integers. Their ergodicity was established and determined by their coefficients. Sufficient and necessary conditions were provided in [4, Theorem 4.1] for ergodicity of perturbed monomials on 2-adic spheres around 1 , by means of a suitable application of van der Put representations. On the other hand, the main tool in [8] was Taylor's formula. By a somehow different approach, a very nice characterization of general 1-Lipschitz measurepreserving functions on the set of 2-adic integers was given in [11, Lemma 3.11 .

In this paper we use a combination of the above mentioned tools with some specific properties of polynomials and their derivatives.

2010 Mathematics Subject Classification: Primary 11S82; Secondary 37P20.

Key words and phrases: ergodic polynomials, transitive functions, 2-adic spheres.

Received 18 January 2017.

Published online 9 March 2017. 
In this section we give a brief description of the notion and properties of 2 -adic numbers and transitive functions.

Let $\mathbb{Q}_{2}$ denote the field of 2-adic numbers endowed with its ultrametric norm $|\cdot|$ and Haar measure $\mu$. It is known that each $x \in \mathbb{Q}_{2}$ has the form $x=\sum_{i=-\infty}^{\infty} x_{i} 2^{i}$, where $x_{i} \in\{0,1\}$ and $\lim _{i \rightarrow-\infty} x_{i}=0$.

Let $K$ be a compact subset of $\mathbb{Q}_{2}$. A function $f: K \rightarrow K$ is measurepreserving on $K$ if and only if $\mu\left(f^{-1}(S)\right)=\mu(S)$ for every measurable subset $S$ of $K$.

A function $f: \mathbb{Z}_{p} \rightarrow \mathbb{Z}_{p}$ is called 1-Lipschitz (or compatible) if

$$
|f(x)-f(y)| \leq|x-y|, \quad \forall x, y \in \mathbb{Z}_{p} .
$$

A description of 1-Lipschitz measure-preserving functions on the compact set of $p$-adic integers was given in [3], [13] and [12.

It is known (see [2, Theorem 1.1] and [3, Proposition 4.26]) that 1Lipschitz measure-preserving functions are bijective and isometric on the set of $p$-adic integers.

A measure-preserving function $f$ is said to be ergodic on some set $E$ if no proper subset of $E$ is invariant under $f$.

A characterization of measure-preserving and ergodic 1-Lipschitz functions on the set of $p$-adic integers was provided in [3, [1], [5, [6], 10].

An invertible measure-preserving function $f: K \rightarrow K$ on some 2-adic compact set $K$ is said to be bijective modulo $2^{n}$ for some integer $n$ if $K$

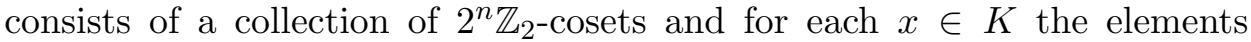
$x, f(x), \ldots, f^{k-1}(x)$ are in distinct $2^{n} \mathbb{Z}_{2}$-cosets, where $k=2^{n} \mu(K)$.

An invertible measure-preserving function $f: K \rightarrow K$ is said to be transitive modulo $2^{n}$ if it is bijective modulo $2^{n}$ and the set $x, f(x), \ldots, f^{k-1}(x)$ is composed of only one cycle. Namely, $\left|f^{k}(x)-x\right| \leq 2^{-n}$, but $\left|f^{r}(x)-x\right|>2^{n}$ for all $r<k$.

In [8, Proposition 4] it was proved that a compatible function on $\mathbb{Z}_{2}$ is isometric if and only if it is measure-preserving and bijective modulo $2^{n}$ for every positive integer $n$. Moreover, in [2, Theorem 1.1], [3, Proposition 4.35] and [8, Theorem 6] it was proved that an isometric function $f$ on $\mathbb{Z}_{2}$ is ergodic if and only if it is transitive modulo $2^{n}$ for every positive integer $n$.

Obviously, these results can be easily generalized to $2^{N} \mathbb{Z}_{2}$-cosets for any integer $N$, so that bijectivity and transitivity are considered modulo $2^{n}$ for every integer $n>N$.

Ergodicity of 1-Lipschitz functions on $p$-adic spheres was studied in [3], [4] and [14] [3, Lemma 4.76] provides necessary and sufficient conditions for ergodicity of 1-Lipschitz functions on $p$-adic spheres. Similar results were obtained in [4] and then applied to ergodic perturbed monomials on 2-adic spheres around the unity (see [4, Theorem 4.1]). [14, Theorem 2.2] gives a 
precise description of ergodic functions on 2-adic spheres centred at arbitrary integers by means of their van der Put representations.

In this work we focus on the behaviour of polynomials with coefficients from $\mathbb{Q}_{2}$ on 2-adic spheres centred at $a=0$. The main result is Theorem 2.5. which gives sufficient conditions for a polynomial on $\mathbb{Q}_{2}$ to be invariant and ergodic on the 2-adic unit sphere. A generalization of this result to other 2 -adic spheres is stated in Theorem 2.6. Although ergodic polynomials with 2 -adic and 3 -adic coefficients were studied in [8], in our study we only focus on the 2-adic case because Lemma 2.4, which is one of our main tools, describes transitivity on the set of 2-adic units. Moreover, Lemma 2.4 is a consequence of [11, Lemma 3.11] for which there is no analogue in general $p$-adic structures.

\section{Ergodic polynomials on 2-adic spheres}

Theorem 2.1. Let $f(x)=\sum_{i=0}^{m} a_{i} x^{i}$ be a polynomial on $\mathbb{Q}_{2}$. Then $f$ is invariant and isometric on $\mathbb{Z}_{2} \backslash 2 \mathbb{Z}_{2}$ if the following conditions are satisfied:

(1) $\left|\sum_{i=0}^{m} a_{i}\right|=1$;

(2) $\left|\sum_{i=1}^{m} i a_{i}\right|=1$;

(3) $\left|\sum_{i=k}^{m}\left(\begin{array}{l}i \\ k\end{array}\right) a_{i}\right| \leq 2^{k-2}$ for all $k \in\{2, \ldots, m\}$.

Proof. We first prove that for all $x \in \mathbb{Z}_{2} \backslash 2 \mathbb{Z}_{2}$ and $k \geq 2$,

$$
\left|\frac{f^{(k)}(x)}{k !}\right| \leq 2^{k-2} \text {. }
$$

Notice that $f^{(k)}(x)=0$ for all $k \geq m+1$, and

$$
f^{(k)}(x)=\sum_{i=k}^{m} i(i-1) \ldots(i-k+1) a_{i} x^{i-k}
$$

for all $k \in\{1, \ldots, m\}$. Hence, from condition (3) we see that (2.1) is true for $x=1$ : indeed, for all $k \in\{2, \ldots, m\}$,

$$
\left|\frac{f^{(k)}(1)}{k !}\right|=\left|\sum_{i=k}^{m} \frac{i(i-1) \ldots(i-k+1)}{k !} a_{i}\right|=\left|\sum_{i=k}^{m}\left(\begin{array}{l}
i \\
k
\end{array}\right) a_{i}\right| \leq 2^{k-2} .
$$

Applying Taylor's formula for $k \in\{2, \ldots, m-1\}$ we get

$$
f^{(k)}(x)-f^{(k)}(1)=\sum_{i=1}^{m-k} \frac{f^{(k+i)}(1)}{i !}(x-1)^{i} .
$$


As mentioned above, for all $i \in\{1, \ldots, m-k\}$, we have

$$
\left|\frac{f^{(k+i)}(1)}{i ! k !}(x-1)^{i}\right| \leq 2^{k+i-2} \frac{(k+i) !}{k ! i !}|x-1|^{i} \leq 2^{k-2} .
$$

Therefore, $\left|\frac{f^{(k)}(x)-f^{(k)}(1)}{k !}\right| \leq 2^{k-2}$, and 2.1 is valid.

The next step is to prove that $\left|f^{\prime}\right|=1$ on $\mathbb{Z}_{2} \backslash 2 \mathbb{Z}_{2}$. Fix $x \in \mathbb{Z}_{2} \backslash 2 \mathbb{Z}_{2}$. Applying Taylor's formula for $f^{\prime}$ gives

$$
f^{\prime}(x)-f^{\prime}(1)=\sum_{i=1}^{m-1} \frac{f^{(i+1)}(x)}{i !}(x-1)^{i} .
$$

Formula 2.1) implies that

$$
\left|\frac{f^{(i+1)}(x)}{i !}(x-1)^{i}\right| \leq\left|(i+1) 2^{i-1}(x-1)^{i}\right| \leq 2^{-1}, \quad \forall i \in\{1, \ldots, m-1\} .
$$

Hence, $\left|f^{\prime}(x)-f^{\prime}(1)\right|<1$. Combining this fact with condition (2) yields $\left|f^{\prime}(x)\right|=1$.

If we apply again Taylor's formula for arbitrary $x, y \in \mathbb{Z}_{2} \backslash 2 \mathbb{Z}_{2}$, we get

$$
f(x)-f(y)=\sum_{i=1}^{m} \frac{f^{(i)}(y)}{i !}(x-y)^{i}=(x-y)\left(\sum_{i=1}^{m} \frac{f^{(i)}(y)}{i !}(x-y)^{i-1}\right) .
$$

From (2.1) it follows that

$$
\left|\frac{f^{(i)}(y)}{i !}(x-y)^{i-1}\right| \leq\left|2^{i-2}(x-y)^{i-1}\right| \leq 2^{-1}, \quad \forall i \in\{2, \ldots, m\},
$$

while $\left|f^{\prime}(y)\right|=1$. Therefore, we must have

$$
|f(x)-f(y)|=|x-y| \text {. }
$$

Combining the fact that $f$ is isometric with condition (1) implies that $f$ is invariant on the set $\mathbb{Z}_{2} \backslash 2 \mathbb{Z}_{2}$.

Lemma 2.2. Let $f$ be a polynomial on $\mathbb{Q}_{2}$. Suppose that $f$ is invariant, isometric and transitive modulo $2^{n}$ on the 2 -adic unit sphere $\mathbb{Z}_{2} \backslash 2 \mathbb{Z}_{2}$, for some $n \geq 2$. Then the following assertions are equivalent:

(1) $f$ is transitive modulo $2^{n+1}$ on $\mathbb{Z}_{2} \backslash 2 \mathbb{Z}_{2}$.

(2) For every $x \in \mathbb{Z}_{2} \backslash 2 \mathbb{Z}_{2}, f^{2^{n-1}}(x) \neq x\left(\bmod 2^{n+1}\right)$ and $\left|\left(f^{2^{n-1}}\right)^{\prime}(x)\right|=1$.

(3) For some $x \in \mathbb{Z}_{2} \backslash 2 \mathbb{Z}_{2}, f^{2^{n-1}}(x) \neq x\left(\bmod 2^{n+1}\right)$ and $\left|\left(f^{2^{n-1}}\right)^{\prime}(x)\right|=1$.

The proof of Lemma 2.2 is similar to the proof of [8, Lemma 8] with some evident modifications. We can just add the fact that since $f$ is isometric, it can be seen that $\left|f^{\prime}\right|=1$ on $\mathbb{Z}_{2} \backslash 2 \mathbb{Z}_{2}$ (see [7, Proposition 2.3]).

The following lemma is a modified version of [8, Proposition 9]. 
Lemma 2.3. Let $f$ be a polynomial on $\mathbb{Q}_{2}$ that satisfies all conditions of Theorem 2.1. Suppose that $f$ is transitive modulo $2^{4}$ on $\mathbb{Z}_{2} \backslash 2 \mathbb{Z}_{2}$. Then $f$ is ergodic on $\mathbb{Z}_{2} \backslash 2 \mathbb{Z}_{2}$.

Proof. It suffices to prove that if for some $s \geq 4, f$ is transitive modulo $2^{s}$, then it is transitive modulo $2^{s+1}$.

We proceed as in the proof of [8, Lemma 8]. For every $x \in \mathbb{Z}_{2} \backslash 2 \mathbb{Z}_{2}$,

$$
\left|\left(f^{2^{s-1}}\right)^{\prime}(x)\right|=\left|\prod_{i=0}^{2^{s-1}-1} f^{\prime}\left(f^{i}(x)\right)\right|=1, \quad f^{0}(x)=x .
$$

From the assumption that $f$ is transitive modulo $2^{s}$ on $\mathbb{Z}_{2} \backslash 2 \mathbb{Z}_{2}$, by Lemma 2.2 we get

$$
\left|f^{2^{s-2}}(x)-x\right|=2^{-s+1} .
$$

Now proceeding as in $[8$, Proposition 9] we find that

$$
\begin{aligned}
f^{2^{s-1}}(x)= & f^{2^{s-2}}\left(f^{2^{s-2}}(x)\right)=f^{2^{s-2}}\left(x+f^{2^{s-2}}(x)-x\right) \\
= & f^{2^{s-2}}(x)+\left(f^{2^{s-2}}\right)^{\prime}(x)\left(f^{2^{s-2}}(x)-x\right) \\
& +\sum_{i=2}^{\infty} \frac{f^{2^{s-2}(i)}(x)}{i !}\left(f^{2^{s-2}}(x)-x\right)^{i} .
\end{aligned}
$$

In this way we obtain

$$
\begin{aligned}
& f^{2^{s-1}}(x)-x=\left(f^{2^{s-2}}(x)-x\right)+\left(f^{2^{s-2}}\right)^{\prime}(x)\left(f^{2^{s-2}}(x)-x\right) \\
& \quad+\sum_{i=2}^{\infty} \frac{f^{2^{s-2}(i)}(x)}{i !}\left(f^{2^{s-2}}(x)-x\right)^{i} \\
& =\left(f^{2^{s-2}}(x)-x\right)\left(1+\left(f^{2^{s-2}}\right)^{\prime}(x)+\sum_{i=2}^{\infty} \frac{f^{2^{s-2}(i)}(x)}{i !}\left(f^{2^{s-2}}(x)-x\right)^{i-1}\right) .
\end{aligned}
$$

It follows that

$$
\begin{aligned}
& \left|f^{2^{s-1}}(x)-x\right| \\
= & \left|f^{2^{s-2}}(x)-x\right| \cdot\left|1+\left(f^{2^{s-2}}\right)^{\prime}(x)+\sum_{i=2}^{\infty} \frac{f^{2^{s-2}(i)}(x)}{i !}\left(f^{2^{s-2}}(x)-x\right)^{i-1}\right| .
\end{aligned}
$$

Recall Faà di Bruno's formula for higher derivatives of composite functions [9]:

$$
(g \circ f)^{(i)}(x)=\sum \frac{i !}{b_{1} ! \ldots b_{i} !} g^{(k)}(f(x)) \prod_{s=1}^{i}\left(\frac{f^{(s)}(x)}{s !}\right)^{b_{s}},
$$

where the sum is taken over different solutions in non-negative integers $b_{1}, \ldots, b_{i}$ of $\sum_{s=1}^{i} s b_{s}=i$ and $k=\sum_{s=1}^{i} b_{s}$. 
By means of this formula we prove that (2.1) is still valid for successive compositions of $f$. Let us first verify that 2.1$)$ is true for $f^{2}$ :

$$
\frac{\left(f^{2}\right)^{(i)}(x)}{i !}=\sum \frac{1}{b_{1} ! \ldots b_{i} !} f^{(k)}(f(x)) \prod_{s=1}^{i}\left(\frac{f^{(s)}(x)}{s !}\right)^{b_{s}} .
$$

We have

$$
\left|\frac{f^{(k)}(f(x))}{b_{1} ! \ldots b_{i} !}\right| \leq\left|\frac{f^{(k)}(f(x))}{k !}\right| \cdot \frac{k !}{b_{1} ! \ldots b_{i} !} \leq 2^{k-2} .
$$

On the other hand,

$$
\begin{aligned}
\left|\prod_{s=1}^{i}\left(\frac{f^{(s)}(x)}{s !}\right)^{b_{s}}\right| & \leq \prod_{s=2}^{i} 2^{(s-2) b_{s}}=2^{\sum_{s=2}^{i} s b_{s}} 2^{-2 \sum_{s=2}^{i} b_{s}} \\
& =2^{i-b_{1}-2 k+2 b_{1}}=2^{i+b_{1}-2 k} .
\end{aligned}
$$

It follows that

$$
\left|\frac{f^{(k)}(f(x))}{b_{1} ! \ldots b_{i} !}\right| \cdot\left|\prod_{s=1}^{i}\left(\frac{f^{(s)}(x)}{s !}\right)^{b_{s}}\right| \leq 2^{i+b_{1}-k-2} \leq 2^{i-2} .
$$

In a similar way, writing $f^{l}$ in the form $f^{l-1} \circ f$ we can deduce the estimate

$$
\left|\frac{\left(f^{l}\right)^{(i)}(x)}{i !}\right| \leq 2^{i-2}
$$

from the assumption that

$$
\left|\frac{\left(f^{k}\right)^{(i)}(x)}{i !}\right| \leq 2^{i-2}
$$

for every integer $k$ greater than 1 and less than $l$.

For every $i \geq 2$ we have

$$
\begin{aligned}
\left|\frac{f^{2^{s-2}(i)}(x)}{i !}\left(f^{2^{s-2}}(x)-x\right)^{i-1}\right| & \leq 2^{i-2}\left|f^{2^{s-2}}(x)-x\right|^{i-1} \\
& =2^{i-2} 2^{(-s+1)(i-1)}=2^{(-s+2)(i-1)-1} \leq 2^{-3} .
\end{aligned}
$$

Moreover, since $s \geq 4$, we have

$\left(f^{2^{s-2}}\right)^{\prime}(x)=\prod_{r=0}^{2^{s-2}-1} f^{\prime}\left(f^{r}(x)\right)=\left(f^{\prime}(x) \cdot f^{\prime}(f(x))\right)^{2^{s-3}}(\bmod 4)=1(\bmod 4)$,

because it can be seen from Taylor's formula that $f^{\prime}$ is 1-Lipschitz, so it is constant $\bmod 4$ on each $2^{2}$-coset of $\mathbb{Z}_{2} \backslash 2 \mathbb{Z}_{2}$.

Therefore,

$$
\left|1+\left(f^{2^{s-2}}\right)^{\prime}(x)\right|=1 / 2,
$$

and (2.2), 2.3) lead to

$$
\left|f^{2^{s-1}}(x)-x\right|=2^{-1}\left|f^{2^{s-2}}(x)-x\right|=2^{-s} .
$$


Lemma 2.4. An isometric function $f: \mathbb{Z}_{2} \backslash 2 \mathbb{Z}_{2} \rightarrow \mathbb{Z}_{2} \backslash 2 \mathbb{Z}_{2}$ is transitive modulo $2^{4}$ if and only if the following conditions are satisfied:

(1) $|f(1)-1|=1 / 2$,

(2) $|f(1)+f(-1)|=1 / 4$,

(3) $|f(1)+f(3)+f(-1)+f(-3)|=1 / 8$.

Proof. Since $f$ is isometric, it can be seen that it is transitive modulo $2^{2}$ if and only if condition (1) is satisfied.

Arguing as in [11, Lemma 3.11] we can prove that $f$ is transitive modulo $2^{n+1}, n \geq 2$, if and only if it is transitive modulo $2^{n}$ and $S_{n}$ is odd, where $S_{n}=\sum_{1 \leq m \leq 2^{n}-1, m \text { odd }} f_{m_{n}}, f(m)=\sum_{i=0}^{\infty} f_{m_{i}} 2^{i}, f_{m_{0}}=1, f_{m_{i}} \in\{0,1\}, \forall i \geq 1$.

Since $f$ is isometric, we get $f_{3_{1}}=f_{(-1)_{1}}$ and $f_{3_{2}} \neq f_{(-1)_{2}}$. Then $S_{2}=$ $f_{1_{2}}+f_{3_{2}}$ is odd if and only if $f_{1_{2}}=f_{(-1)_{2}}$. We immediately see that this is equivalent to (2), because isometricity implies that $f_{1_{1}} \neq f_{(-1)_{1}}$. In a similar way it suffices to prove that if $f$ is transitive modulo $2^{3}$ then condition (3) is equivalent to $S_{3}$ being odd.

First by isometry and condition (1) we have

$$
f_{(-1)_{1}}+f_{(-3)_{1}}=f_{(1)_{1}}+f_{(3)_{1}}=1 ;
$$

then by isometricity and the fact that $S_{2}$ is odd we get

$$
f_{(-1)_{2}}+f_{(-3)_{2}}=f_{(1)_{2}}+f_{(3)_{2}}=1 .
$$

Hence,

$$
\begin{aligned}
f(1) & +f(3)+f(-1)+f(-3) \\
= & 4+2 \cdot 2+2 \cdot 2^{2}+\sum_{i=3}^{\infty}\left(f_{1_{i}}+f_{3_{i}}+f_{(-3)_{i}}+f_{(-1)_{i}}\right) 2^{i} \\
= & 2^{4}+\left(f_{1_{3}}+f_{3_{3}}+f_{(-3)_{3}}+f_{(-1)_{3}}\right) 2^{3}+\sum_{i=4}^{\infty}\left(f_{1_{i}}+f_{3_{i}}+f_{(-3)_{i}}+f_{(-1)_{i}}\right) 2^{i} . \\
= & \left(f_{1_{3}}+f_{3_{3}}+f_{(7)_{3}}+f_{(5)_{3}}\right) 2^{3}+2^{3}\left(f_{(-1)_{3}}-f_{7_{3}}+f_{(-3)_{3}}-f_{5_{3}}\right) \\
& +2^{4}+\sum_{i=4}^{\infty}\left(f_{1_{i}}+f_{3_{i}}+f_{(-3)_{i}}+f_{(-1)_{i}}\right) 2^{i} .
\end{aligned}
$$

Since $f_{(-1)_{3}} \neq f_{7_{3}}$ and $f_{(-3)_{3}} \neq f_{5_{3}}$, we see that $f_{(-1)_{3}}-f_{7_{3}}+f_{(-3)_{3}}-f_{5_{3}}$ is even. Therefore, $S_{3}=f_{1_{3}}+f_{3_{3}}+f_{5_{3}}+f_{7_{3}}$ is odd if and only if condition (3) is satisfied.

Theorem 2.5. Let $f(x)=\sum_{i=0}^{m} a_{i} x^{i}$ be a polynomial on $\mathbb{Q}_{2}$. Then $f$ is invariant, isometric and ergodic on $\mathbb{Z}_{2} \backslash 2 \mathbb{Z}_{2}$ if the following assumptions are satisfied: 
(1) $\left|\sum_{i=0}^{m} a_{i}-1\right|=1 / 2 ;$
(2) $\left|\sum_{0 \leq i \leq m, i \text { even }} a_{i}\right|=1 / 2 ;$
(3) $\left|\sum_{i=1}^{m} i a_{i}\right|=1 ;$
(4) $\left|\sum_{i=s}^{m}\left(\begin{array}{l}i \\ s\end{array}\right) a_{i}\right| \leq 2^{s-2}$ for all $s \in\{2, \ldots, m\} ;$
(5) $\left|\sum_{s=1}^{m} 2^{s} \sum_{s \leq i \leq m, i \text { even }}\left(\begin{array}{l}i \\ s\end{array}\right) a_{i}\right| \leq 1 / 8$ for all $s \in\{2, \ldots, m\}$.

Proof. Using Lemma 2.3 it suffices to verify that all conditions of Theorem 2.1 and Lemma 2.4 are satisfied. The first assumption in the present theorem yields condition (1) of Theorem 2.1 and condition (1) of Lemma 2.4. Assumption (2) is just a modified form of condition (2) in Lemma 2.4. Assumptions (3) and (4) are precisely conditions (2) and (3) of Theorem 2.1.

Let us verify that assumption (5) combined with (2) gives condition (3) of Lemma 2.4:

$$
\begin{aligned}
f(1)+f(3)+f(-1)+f(-3) & =2 \sum_{0 \leq i \leq m, i \text { even }} a_{i}+2 \sum_{0 \leq i \leq m, i \text { even }} 3^{i} a_{i} \\
& =2 \sum_{0 \leq i \leq m, i \text { even }} a_{i}+2 \sum_{0 \leq i \leq m, i \text { even }} \sum_{s=0}^{i} 2^{s}\left(\begin{array}{l}
i \\
s
\end{array}\right) a_{i} \\
& =2 \sum_{0 \leq i \leq m, i \text { even }} a_{i}+2 \sum_{s=0}^{m} 2^{s} \sum_{s \leq i \leq m, i \text { even }}\left(\begin{array}{l}
i \\
s
\end{array}\right) a_{i} \\
& =4 \sum_{0 \leq i \leq m, i \text { even }} a_{i}+2 \sum_{s=1}^{m} 2^{s} \sum_{s \leq i \leq m, i \text { even }}\left(\begin{array}{l}
i \\
s
\end{array}\right) a_{i} .
\end{aligned}
$$

Applying assumption (2) we get

$$
\left|4 \sum_{0 \leq i \leq m, i \text { even }} a_{i}\right|=\frac{1}{8}
$$

Hence from assumption (5) we obtain

$$
|f(1)+f(3)+f(-1)+f(-3)|=\left|4 \sum_{0 \leq i \leq m, i \text { even }} a_{i}\right|=\frac{1}{8} .
$$

Theorem 2.6. Let $g(x)=\sum_{i=0}^{m} A_{i} x^{i}$ be a polynomial on $\mathbb{Q}_{2}$. Then $g$ is invariant, isometric and ergodic on $2^{-N} \mathbb{Z}_{2} \backslash 2^{-N+1} \mathbb{Z}_{2}$ if the following 
assumptions are satisfied:

(1) $\left|\sum_{i=0}^{m} 2^{-N(i-1)} A_{i}-1\right|=1 / 2 ;$
(2) $\left|\sum_{0 \leq i \leq m, i \text { even }} 2^{-N(i-1)} A_{i}\right|=1 / 2 ;$
(3) $\left|\sum_{i=1}^{m} i 2^{-N(i-1)} A_{i}\right|=1 ;$
(4) $\left|\sum_{i=s}^{m}\left(\begin{array}{l}i \\ s\end{array}\right) 2^{-N(i-1)} A_{i}\right| \leq 2^{s-2}$ for all $s \in\{2, \ldots, m\} ;$
(5) $\left|\sum_{s=1}^{m} 2^{s} \sum_{s \leq i \leq m, i \text { even }}\left(\begin{array}{l}i \\ s\end{array}\right) 2^{-N(i-1)} A_{i}\right| \leq 1 / 8$ for all $s \in\{2, \ldots, m\}$.

Proof. Let $f(x)=\sum_{i=0}^{m} a_{i} x^{i}$, where $a_{i}=2^{-N(i-1)} A_{i}$ for every $i \in$ $\{0, \ldots, m\}$. It is easily seen that $f$ satisfies all conditions of Theorem 2.5. Moreover, the relation $g\left(2^{-N} x\right)=2^{-N} f(x)$ shows that $g$ and $f$ are simultaneously invariant, isometric and ergodic on respectively the spheres $2^{-N} \mathbb{Z}_{2} \backslash 2^{-N+1} \mathbb{Z}_{2}$ and $\mathbb{Z}_{2} \backslash 2 \mathbb{Z}_{2}$

Acknowledgements. The author would like to thank the referee for valuable suggestions.

\section{References}

[1] V. Anashin, Ergodic transformations in the space of p-adic integers, in: $p$-Adic Mathematical Physics, AIP Conf. Proc. 826, Amer. Inst. Phys., Melville, NY, 2006, 3-24.

[2] V. S. Anashin, Uniformly distributed sequences of p-adic integers, Discrete Math. Appl. 12 (2002), 527-590.

[3] V. Anashin and A. Khrennikov, Applied Algebraic Dynamics, de Gruyter Exp. Math. 49, de Gruyter, Berlin, 2009.

[4] V. Anashin, A. Khrennikov and E. Yurova, Ergodicity criteria for non-expanding transformations of 2-adic spheres, Discrete Contin. Dynam. Systems 34 (2014), 367377 .

[5] J. Bryk and C. E. Silva, Measurable dynamics of simple p-adic polynomials, Amer. Math. Monthly 112 (3) (2005), 212-232.

[6] Z. Coelho and W. Parry, Ergodicity of p-adic multiplications and the distribution of Fibonacci numbers, in: Topology, Ergodic Theory, Real Algebraic Geometry, Amer. Math. Soc. Transl. (2) 202 (2001), 51-70.

[7] H. Diao and C. E. Silva, Digraph representations of rational functions over the p-adic numbers, p-adic Numbers Ultrametric Anal. Appl. 3 (2011), 23-38.

[8] F. Durand and F. Paccaut, Minimal polynomial dynamics on the set of 3-adic integers, Bull. London Math. Soc. 41 (2009), 302-314.

[9] F. Faà di Bruno, Note sur une nouvelle formule de calcul différentiel, Quart. J. Pure Appl. Math. 1 (1857), 359-360. 
[10] M. Gundlach, A. Khrennikov and K.-O. Lindahl, On ergodic behavior of p-adic dynamical systems, Infin. Dimens. Anal. Quantum Probab. Related Topics 4 (2001), 569-577.

[11] S. Jeong, Toward the ergodicity of p-adic 1-Lipschitz functions represented by the van der Put series, J. Number Theory 133 (2013), 2874-2891.

[12] A. Khrennikov and E. Yurova, Criteria of measure-preserving for p-adic dynamical systems in terms of the van der Put basis, J. Number Theory 133 (2013), 484-491.

[13] E. Yurova, On measure-preserving functions over $\mathbb{Z}_{3}, p$-adic Numbers Ultrametric Anal. Appl. 4 (2012), 326-335.

[14] E. Yurova, The ergodicity of 1-Lipschitz transformations on 2-adic spheres, in: Valuation Theory in Interaction, EMS Ser. Congr. Rep., Eur. Math. Soc., Zürich, 2014, 596-599.

Nacima Memić

Department of Mathematics

Faculty of Natural Sciences and Mathematics

University of Sarajevo

Zmaja od Bosne 33-35

Sarajevo, Bosnia and Herzegovina

E-mail: nacima.o@gmail.com 